\title{
Religiosidade e qualidade de vida relacionada à saúde dos idosos em um município na Bahia, Brasil
}

\author{
Religiosity and health-related quality of life of elderly in a city in Bahia, Brazil
}

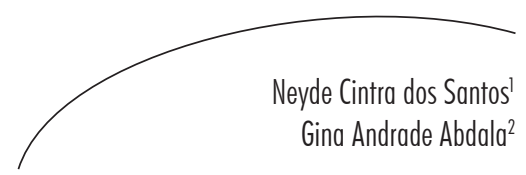

Resumo

Objetivo: Avaliar as dimensões da qualidade de vida relacionada à saúde em idosos da comunidade e sua relação com a religiosidade organizacional (RO), religiosidade não organizacional (RNO) e religiosidade intrínseca (RI). Método: Estudo transversal de abordagem quantitativa, com amostra aleatória composta por 82 idosos cadastrados na Estratégia de Saúde da Família de Capoeiruçu, bairro do município de CachoeiraBA, Brasil. Resultados: O sexo feminino predominou, com 61,0\%. A idade média foi (71 $\pm 9,39)$. Nas oito dimensões da qualidade de vida, o escore variou entre 64,3 a 77,3. A RO foi $74,4 \%$ e a RNO foi de $89,1 \%$. Para as questões sobre RI: sentir a presença de Deus, agir de acordo com suas crenças e se eles se esforçam para viver a religião em todos os aspectos da vida, encontraram-se percentuais de 95\%, 90,2\% e 84,2\% respectivamente. Usando o teste do qui-quadrado, verificou-se que as "limitações por aspectos emocionais" foi a dimensão que recebeu maior influência de $\mathrm{RO}\left(\mathrm{X}^{2}=11,539\right.$, $\mathrm{p}=0,001, \mathrm{~V}$ de Cramer=0,372), RNO $\left(\mathrm{X}^{2}=7,949, \mathrm{p}=0,005, \mathrm{~V}\right.$ de Cramer=0,309) e RI $\left(\mathrm{X}^{2}=5,126, \mathrm{p}=0,05, \mathrm{~V}\right.$ de Cramer $\left.=0,249\right)$. Constatou-se também influência positiva sobre as dimensões "limitações por aspectos físicos, dor, estado geral de saúde, saúde mental e social". Nenhuma associação foi encontrada entre religiosidade e as dimensões "capacidade funcional” e "vitalidade”. Conclusões: Infere-se que há associação positiva entre religiosidade nas três dimensões (RO, RNO e RI) e a qualidade de vida relacionada à saúde dos idosos.

\section{Abstract}

Objective: To evaluate the dimensions of health-related quality of life in older adults and its relationship with organizational religious affiliation (ORA), non-organizational religious affiliation (NORA) and intrinsic religiosity (IR). Method: Cross-sectional study with quantitative approach, with a random sample of 82 elderly enrolled in the Family Health Strategy in Capoeiruçu, Cachoeira-BA, Brazil. Results: The female dominated with $61.4 \%$. The mean age was $(71 \pm 9.39)$. In the eight dimensions of quality of life, the score

\footnotetext{
Curso de Pós-graduação em Saúde Pública. Faculdade Adventista da Bahia, Cachoeira, BA, Brasil.

2 Programa de Pós-graduação em Promoção da Saúde. Centro Universitário Adventista de São Paulo. São Paulo, SP, Brasil.
}

Palavras-chave: Religião. Espiritualidade. Idosos. Qualidade de vida. 
ranged from 64.3 to 77.3 . ORA was $74.4 \%$; NORA was 89.1. For questions about IR: feel the presence of God, act according to their beliefs and if they strive to live the religion in all aspects of life, percentages of $95 \%, 90.2 \%$ and $84.2 \%$ were respectively found. Using the chi-square test, it was found that the dimension "limitations due to emotional problems" received the greatest influence of ORA $\left(\mathrm{X}^{2}=11.539\right.$; $\mathrm{p}=0.001$; Cramer's $\mathrm{V}=$ 0.372), NORA $\left(X^{2}=7.949 ; p=0.005\right.$; Cramer's $\left.V=0.309\right)$ and $\operatorname{IR}\left(X^{2}=5.126 ; p=0.05\right.$; Cramer's $V=0.249)$. It was also found a positive influence on the limitations due to physical, bodily pain, general health, mental and social health. No association was found between religiosity and "functional capacity" and "vitality" dimensions. Conclusions: It is inferred that there is a positive association between religiosity in its three dimensions (ORA, NORA and IR) and health-related quality of life for the elderly.
Key words: Religion. Spirituality. Elderly. Quality of life.

\section{INTRODUÇÃO}

Para o idoso, as questões relacionadas à religiosidade têm significado muito especial, principalmente entre aqueles que vivenciam problemas ou alguma situação dolorosa, seja por doença ou pelas consequências deixadas por ela ${ }^{1}$. As mudanças físicas, psicológicas e sociais, que são comuns aos idosos, acarretam situações de perdas, declínio da saúde, afastamento do mercado de trabalho e eventos não controláveis, cujo enfrentamento de modo efetivo pode ser alcançado através das crenças espirituais e práticas religiosas. ${ }^{2,3}$

O envolvimento religioso ou religiosidade compreende os comportamentos, atitudes, valores, crenças, sentimentos e experiências motivados pelo contexto religioso, ${ }^{4}$ desdobrandose em três dimensões: religiosidade organizacional $(\mathrm{RO})$, religiosidade não organizacional $(\mathrm{RNO}) \mathrm{e}$ religiosidade intrínseca (RI). ${ }^{4,5}$

A RO compreende os comportamentos religiosos que ocorrem no contexto da instituição religiosa (como a frequência às atividades religiosas formais) e o desempenho de cargos ou funções religiosas. ${ }^{6}$ Já a RNO engloba os comportamentos religiosos privados ou informais, isto é, que ocorrem fora do contexto da instituição religiosa, sem local e tempo fixos e sem seguirem formas litúrgicas preestabelecidas, podendo se manifestar individualmente ou em pequenos grupos familiares e informais. ${ }^{6}$

Finalmente, a RI avalia o quanto a religião pode motivar ou influenciar comportamentos, decisões e, de forma geral, a vida do sujeito. Trata-se de uma dimensão subjetiva, de quanto ou como o indivíduo percebe a importância da religião em sua vida. ${ }^{5}$

A necessidade espiritual se apresenta mais aguçada no idoso devido às características de sua existência, não excluindo as necessidades do ser humano em todas as suas fases. Segundo Mickley \& Carson (1995 apud Lindolpho et al.), ${ }^{3}$ pessoas acima de 65 anos possuem mais comportamentos e atitudes religiosas do que as pessoas mais jovens, evidenciando, desse modo, a importância da espiritualidade nesta fase da vida. No estudo de Araújo, ${ }^{8}$ boa parcela dos idosos atribuiu importância à religiosidade para a melhoria de sua condição, ajudando-os na integração social e psicológica, fator fundamental para promover a qualidade de vida e bem-estar. Para muitas pessoas, a espiritualidade/religiosidade é uma fonte de conforto, significado e força, estando relacionada ao bem-estar físico, mental e à busca de um propósito de vida. ${ }^{9}$

Segundo Saad \& Medeiros, ${ }^{10} \quad[. .$.$] \quad a$ espiritualidade proporciona crescimento nos vários campos do relacionamento: no campo intrapessoal (consigo próprio); gera esperança, altruísmo e idealismo, além de dar propósito para a vida e para o sofrimento; no campo interpessoal (com os outros) gera tolerância, unidade e o senso de pertencer a um grupo; no campo transpessoal (com um poder supremo), desperta o amor incondicional, adoração e crença de não estar só.” 
Em geral, os adeptos das crenças religiosas apresentam menor probabilidade de riscos comportamentais. Abdala et al. ${ }^{11}$ afirmam que as pessoas religiosas tendem a evitar comportamentos nocivos que aumentam os riscos de doenças e de morte.

A qualidade de vida na velhice, segundo o Estatuto do Idoso, ${ }^{12}$ implica em garantir assistência à saúde, liberdade de escolha, amigos, moradia, conforto material, imagem corporal, aparência, sentir-se fisicamente bem, autoestima, sentimentos positivos, relações interpessoais, suporte social, participação em atividades físicas e recreativas, sexualidade, espiritualidade e crenças.

O construto "qualidade de vida relacionada à saúde" (QVRS) incorpora as experiências das pessoas diante de questões mais diretamente ligadas ao processo saúde-doença e, nesse sentido, amplia a noção tradicional de saúde embasada principalmente em indicadores de morbidade e mortalidade. Inclui aspectos da qualidade de vida geral, como o estado de saúde, a capacidade funcional e o apoio social, que afetam tanto a saúde física como a mental. ${ }^{13}$

No estudo de Cruz, ${ }^{14}$ a QVRS para o idoso esteve associada à idade, ao sexo, à escolaridade, ao número de medicamentos, a ter paz/ tranquilidade e possuir uma religião. Também esteve associada às dificuldades para as atividades básicas e instrumentais de vida diária.

Assim, o objetivo deste estudo foi avaliar as dimensões da qualidade de vida relacionada à saúde em idosos da comunidade e sua relação com a religiosidade organizacional (RO), religiosidade não organizacional (RNO) e religiosidade intrínseca (RI).

\section{METODOLOGIA}

Trata-se de estudo descritivo, exploratório, de corte transversal, quantitativo, tipo pesquisa de campo, cuja principal finalidade é "conseguir informações acerca de um problema para o qual se procura uma resposta, ou de uma hipótese que se queira comprovar, ou ainda descobrir novos fenômenos ou relações". ${ }^{15}$

Esse tipo de estudo se adéqua a esta pesquisa, por utilizar métodos formais, caracterizados pela precisão e controle estatísticos, com a finalidade de fornecer dados para a verificação de hipóteses e definir criteriosamente a amostra que foi estudada.

O estudo foi realizado em Capoeiruçu, bairro do município de Cachoeira, estado da Bahia, Brasil. A população de estudo foi composta por 265 idosos, com 60 anos ou mais, cadastrados na Estratégia de Saúde da Família (ESF) de Capoeiruçu, sendo 144 do sexo feminino e 121 do masculino. A amostra aleatória de 90 idosos foi elaborada com um nível de confiança de $95 \%$ e a margem de erro de $8,54 \%$. Proporcionalmente, para o sexo feminino foram sorteadas 49 mulheres idosas e 41 homens. Oito deles foram considerados perdidos por não terem respondido completamente o instrumento de QVRS, totalizando 82 idosos (48 mulheres e 34 homens). Todos que aceitaram participar da pesquisa assinaram o Termo de Consentimento Livre e Esclarecido (TCLE).

A coleta de dados aconteceu nos meses de maio e junho de 2011 e foram utilizados como instrumentos de pesquisa, o Índice de Religiosidade de Duke (Duke-Durel), validado no Brasil por Taunay et al. ${ }^{16}$ e desenvolvido por Koenig, Meador \& Parkerson. ${ }^{17}$ Esse instrumento possui cinco itens que captam três das dimensões de religiosidade que mais se relacionam com desfechos em saúde dos indivíduos: RO, RNO e RI. Os primeiros dois itens abordam a RO e a RNO embasados em estudos epidemiológicos realizados nos Estados Unidos, relacionados a indicadores de saúde física, mental e suporte social. Os outros itens referem-se à RI e foram extraídos de um questionário de religiosidade intrínseca de Allport \& Ross. ${ }^{18}$

Outro instrumento utilizado foi o Short Form-36 (SF-36), que mede a qualidade de vida e foi desenvolvido no final dos anos 1980 nos Estados Unidos. O SF-36 foi traduzido, 
adaptado e validado para a cultura brasileira, sendo utilizado para avaliar a qualidade de vida tanto da população geral quanto de idosos. ${ }^{19}$ É um instrumento de fácil acesso, possível de ser utilizado em diferentes populações e faixas etárias, inclusive em idosos. Seu objetivo é avaliar a qualidade de vida do idoso dentro dos parâmetros da velhice saudável, considerando o contexto e as preferências do idoso. ${ }^{20,21}$

O SF-36 é composto por 11 questões e 36 itens que englobam oito componentes (domínios ou dimensões), representados por capacidade funcional (dez itens); limitação por aspectos físicos (quatro itens); dor (dois itens); estado geral da saúde (cinco itens); vitalidade (quatro itens); aspectos sociais (dois itens); limitação por aspectos emocionais (três itens); saúde mental (cinco itens); e uma questão comparativa sobre a percepção atual da saúde há um ano. Para obter os escores, foram feitos os cálculos de cada domínio do SF-36. Cada participante do estudo recebeu um escore de $00-100$, relacionado às oito dimensões da qualidade de vida do instrumento SF-36, sendo que quanto mais elevado o escore, melhor o índice da Qualidade de Vida (QV). ${ }^{19}$
A análise dos dados foi realizada através do programa estatístico SPSS, versão 17. O estudo foi aprovado pelo Comitê de Ética em Pesquisa das Faculdades Adventista da Bahia, sob número CAAE 0044.0.070.000-11.

\section{RESULTADOS}

A idade dos entrevistados variou entre $60 \mathrm{e}$ 96 anos, com média de 71 anos e desvio padrão de 9,39. Predominantemente, a maioria estava na categoria de 60-69 anos (50,6\%) e sexo feminino $(58,5 \%)$. A religião predominante foi a católica, com um total de 50 adeptos (60,2\%); seguida pela evangélica, com 22 (27,7\%); sete $(8,4 \%)$ agnósticos; e três $(3,6 \%)$ não relataram.

Pode-se perceber que a maioria $(37,8 \%)$ dos idosos da ESF de Capoeiruçu (Cachoeira-BA) respondeu que comparece a igreja, templo ou algum encontro religioso mais que uma vez por semana; $19,5 \%$ frequentam uma vez na semana; $17,1, \%$ duas ou três vezes por mês; e 12,2\%, algumas vezes por ano. Aqueles que vão à igreja uma vez por ano ou menos são $12,2 \%$ e os que nunca vão representam $1,2 \%$ (tabela 1$)$.

Tabela 1. Perfil dos idosos quanto à Religiosidade Organizacional (RO). Cachoeira, BA, 2011.

Com que frequência você vai a uma igreja, templo ou outro encontro religioso

Respostas

\begin{tabular}{lcc}
\multicolumn{1}{c}{ Respostas } & $\mathrm{n}$ & $\%$ \\
\cline { 2 - 3 } & & 37,8 \\
Mais do que uma vez por semana & 31 & 19,5 \\
Uma vez por semana & 16 & 17,1 \\
Duas ou três vezes por mês & 14 & 12,2 \\
Algumas vezes por ano & 10 & 12,2 \\
Uma vez por ano ou menos & 10 & 1,2 \\
Nunca & 1 & 100,0 \\
Total & 82 & \\
\hline
\end{tabular}

Fonte- Pesquisa de campo. Cachoeira - BA, 2011. 
Ao serem indagadas sobre a frequência com que dedicam seu tempo a atividades religiosas, como preces, rezas, meditações, leitura da Bíblia ou de outros textos religiosos, isto é, a RNO, $43,9 \%$ disseram realizar essas atividades mais do que uma vez ao dia; $35,4 \%$ realizam-nas diariamente; $9,8 \%$ uma vez por semana; $3,7 \%$ uma vez por mês; $2,4 \%$ poucas vezes por mês e $4,9 \%$ raramente ou nunca (tabela 2 ).

Tabela 2. Perfil dos idosos segundo a Religiosidade Não Organizacional (RNO). Cachoeira, BA, 2011.

\begin{tabular}{lcc}
\hline \multirow{2}{*}{ Respostas } & \multicolumn{2}{c}{$\begin{array}{c}\text { Com que frequência dedica o seu tempo a atividades } \\
\text { religiosas individuais, como preces, rezas, meditações, } \\
\text { leitura da Bíblia ou de outros textos religiosos }\end{array}$} \\
\cline { 2 - 3 } Mais do que uma vez ao dia & $\mathrm{n}$ & $\%$ \\
Diariamente & 36 & 43,9 \\
Duas ou mais vezes por semana & 29 & 35,4 \\
Uma vez por semana & 8 & 9,8 \\
Poucas vezes por mês & 3 & 3,7 \\
Raramente ou nunca & 2 & 2,4 \\
Total & 4 & 4,9 \\
\hline
\end{tabular}

Fonte- Pesquisa de campo. Cachoeira - BA, 2011.

Quando foram questionados sobre a RI, $61,7 \%$ disseram sentir a presença de Deus ou do Espírito Santo em suas vidas; 33,3\% afirmaram que em geral é verdade e $3,7 \%$ que não estão certos; $1,2 \%$ disse que em geral não é verdade e $1,2 \%$ afirmou não ser verdade.

Quando foram questionados "se suas crenças religiosas estão realmente por trás de toda sua maneira de viver", 52,4\% responderam que isto era totalmente verdade para eles; 37,8\% disseram que em geral era verdade; $8,5 \%$ não estavam certos e 1,2\% disse que em geral não era verdade. Na terceira pergunta da RI, 48,8\% disseram que a afirmação "eu me esforço muito para viver a minha religião em todos os aspectos da minha vida" era totalmente verdade na vida, enquanto que $35,4 \%$ responderam que "em geral é verdade"; $12,2 \%$ não estavam certos; $2,4 \%$ afirmaram que "em geral não é verdade"; e 1,2\% afirmou que "não é verdade" (tabela 3). 
Tabela 3. Perfil dos idosos segundo a Religiosidade Intrínseca (RI). Cachoeira, BA, 2011.

\begin{tabular}{lcccccc}
\hline Respostas & $\begin{array}{c}\text { Em minha vida eu } \\
\text { sinto a presença de } \\
\text { Deus ou do } \\
\text { Espírito Santo }\end{array}$ & $\begin{array}{c}\text { As minhas crenças } \\
\text { religiosas estão realmente } \\
\text { por trás de toda minha } \\
\text { maneira de viver }\end{array}$ & $\begin{array}{c}\text { Eu me esforço muito para } \\
\text { viver a minha religião } \\
\text { em todos os aspectos da } \\
\text { minha vida }\end{array}$ \\
\hline Totalmente verdade & $\mathrm{n}$ & $\%$ & $\mathrm{n}$ & $\%$ & $\mathrm{n}$ & $\%$ \\
Em geral é verdade & 50 & 61,7 & 43 & 52,4 & 40 & 48,8 \\
Não estou certo & 3 & 33,3 & 31 & 37,8 & 29 & 35,4 \\
Em geral não é verdade & 1 & 3,7 & 07 & 8,5 & 10 & 12,2 \\
Não é verdade & 1 & 1,2 & 01 & 1,2 & 2 & 2,4 \\
Total & 1,2 & 0 & 0 & 1 & 1,2 \\
\hline
\end{tabular}

Fonte- Pesquisa de campo. Cachoeira - BA, 2011.

Quanto aos resultados do SF-36, segundo as oito dimensões, no item "capacidade funcional", $67(81,7 \%)$ obtiveram um escore maior que 50 e apenas 15 (18,3\%) obtiveram um escore menor que 50. Em relação aos aspectos físicos $51(61,4 \%)$ obtiveram o escore maior que 50 e $32(38,6 \%)$ menores que 50. Ao item "dor", 58 $(70,7 \%)$ tiveram o escore maior que 50 e 24 $(29,3 \%)$ menores que 50.

Relacionados à saúde geral, 74,5\% tiveram o escore maior que 50 e $25,3 \%$ menores que 50 . Em "vitalidade", 74,7\% obtiveram o escore maior que 50 e 25,3\% menores que 50. Quanto aos aspectos sociais, $75,9 \%$ tiveram o escore maior que 50 e $24,1 \%$ menores que 50 . Nos aspectos emocionais, $69(83,1 \%)$ tiveram o escore maior que 50 e $14(16,9 \%)$ menores que 50.

Em relação aos escores de saúde mental, 72 $(83,8 \%)$ obtiveram nota maior que 50 e somente $10(12,2 \%)$ menor que 50 .

Os idosos da ESF de Capoeiruçu apresentam média acima de $50 \mathrm{em}$ todos os domínios de QV, sendo que "vitalidade" e "aspectos físicos" obtiveram menor nota e "aspectos emocionais" e "saúde mental" obtiveram maior nota (figura 1). 


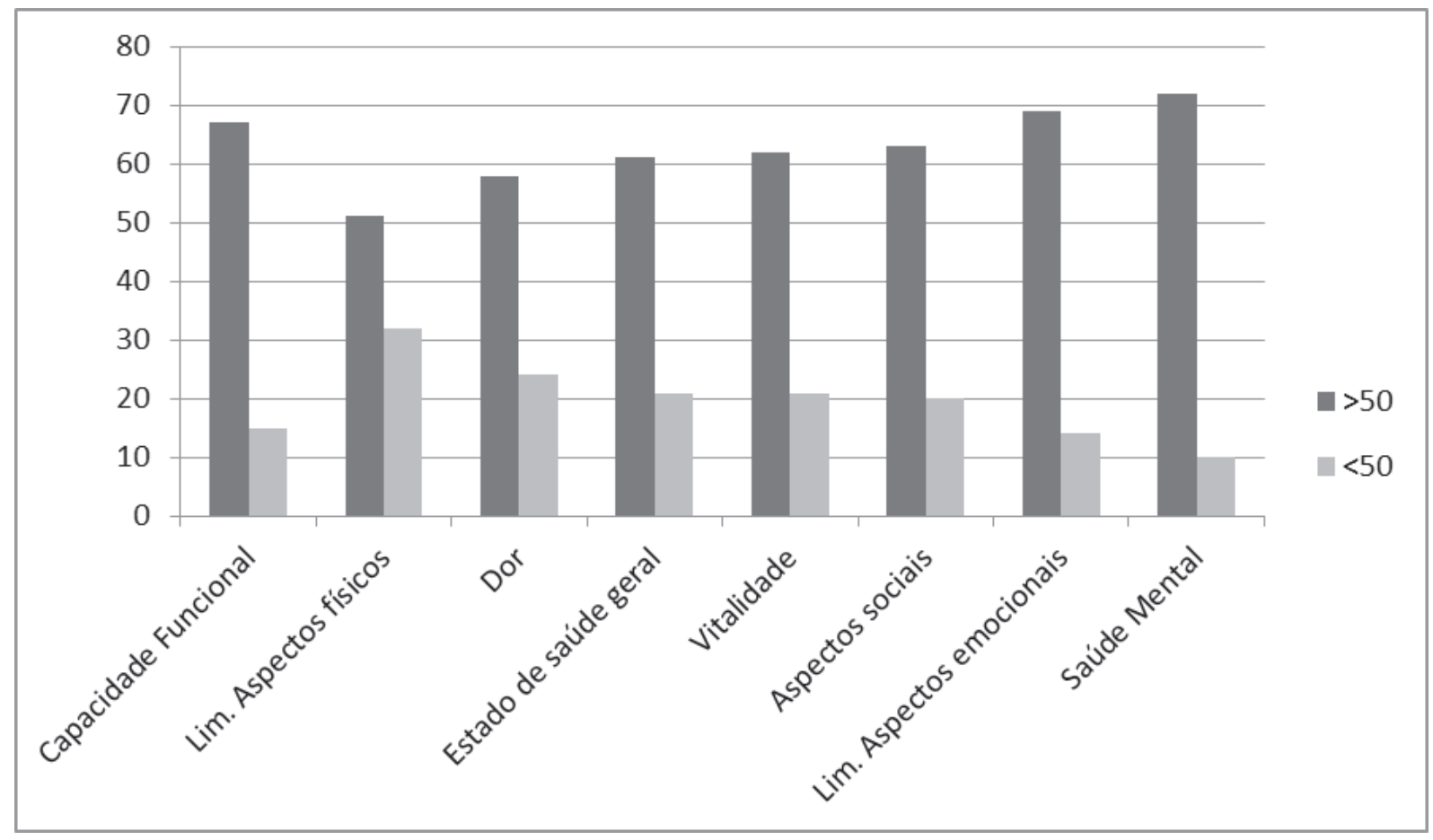

Figura 1. Distribuição das dimensões da QVRS expressa por escores $>50$ e $<50$. Cachoeira-BA, 2011.

Fonte- Pesquisa de campo. Cachoeira - BA, 2011.

$\mathrm{Na}$ tabela 4, foi apresentada uma associação utilizando o teste do qui-quadrado entre os níveis de religiosidade e os domínios de qualidade de vida. Constatou-se que as limitações por aspectos emocionais (LAE) foi a dimensão que esteve mais associada à $\mathrm{RO}\left(\mathrm{X}^{2}=11,534 ; \mathrm{p}=0,001 ; \mathrm{V}\right.$ de Cramer $=0,373)$; ̀̀ $\mathrm{RNO}\left(\mathrm{X}^{2}=7,949 ; \mathrm{p}=\right.$ $0,005 ; \mathrm{V}$ de Cramer $=0,309)$ e RI $\left(\mathrm{X}^{2}=5,126 ; \mathrm{p}\right.$ $=0,05, \mathrm{~V}$ de Cramer $=0,249)$. Isto significa que, elevando-se o $\mathrm{V}$ de Cramer ao quadrado (medida de efeito), $14 \%, 10 \%$ e $6 \%$, respectivamente, dessas variações dentre aqueles que obtiveram escores de QV maiores que 50 estavam entre aqueles que frequentavam a igreja duas ou mais vezes por semana, são regulares nas atividades religiosas privadas e se esforçam para viver sua religião em todos os aspectos da vida. Também foi encontrada associação positiva sobre as dimensões "limitações por aspectos físicos", "dor", "saúde geral", "aspectos sociais" e "saúde mental". Por outro lado, nenhuma associação foi encontrada entre os níveis de religiosidade e as dimensões "capacidade funcional" e "vitalidade" (tabela 4). 
Tabela 4. Distribuição do $\mathrm{X}^{2}$, valor de p e coeficiente de V de Cramer das dimensões da qualidade de vida e a religiosidade (somente os resultados com p significante). Cachoeira, BA, 2011.

\begin{tabular}{|c|c|c|c|c|c|c|c|}
\hline \multirow[b]{2}{*}{$\begin{array}{l}\text { Medidas de } \\
\text { religiosidade }\end{array}$} & \multirow[b]{2}{*}{$\begin{array}{c}\text { Teste } \\
\text { estatístico }\end{array}$} & \multicolumn{6}{|c|}{ Dimensões da qualidade de vida } \\
\hline & & LAF & Dor & SG & AS & LAE & SM \\
\hline \multirow[t]{3}{*}{$\mathrm{RO}$} & $\mathrm{X}^{2}$ & & & & 4,448 & 11,534 & \\
\hline & $\mathrm{p}$ & & & & 0,035 & 0,01 & \\
\hline & Cramer's V & & & & 0,231 & 0,373 & \\
\hline \multirow[t]{3}{*}{ RNO } & $\mathrm{X}^{2}$ & 4,937 & 4,788 & & & 7,949 & \\
\hline & $\mathrm{p}$ & 0,026 & 0,029 & & & 0,005 & \\
\hline & Cramer's V & 0,244 & 0,242 & & & 0,309 & \\
\hline \multirow{3}{*}{$\begin{array}{l}\text { RI- sente a presença } \\
\text { de Deus }\end{array}$} & $\mathrm{X}^{2}$ & & & 8,114 & & & 11,364 \\
\hline & $\mathrm{p}$ & & & 0,04 & & & 0,01 \\
\hline & Fisher & & & $0,015^{*}$ & & & $0,012^{*}$ \\
\hline \multirow{3}{*}{$\begin{array}{l}\text { RI- crenças por trás } \\
\text { da maneira de viver }\end{array}$} & $\mathrm{X}^{2}$ & & & 8,945 & & & 9,998 \\
\hline & $\mathrm{p}$ & & & 0,003 & & & 0,02 \\
\hline & Cramer's V & & & 0,330 & & & $0,010^{*}$ \\
\hline \multirow{3}{*}{$\begin{array}{l}\text { RI- esforço para viver } \\
\text { a religião em todos os } \\
\text { aspectos vida }\end{array}$} & $\mathrm{X}^{2}$ & & & & & 5,126 & 5,098 \\
\hline & $\mathrm{p}$ & & & & & 0,05 & 0,02 \\
\hline & Cramer's V & & & & & 0,249 & 0,24 \\
\hline
\end{tabular}

$\mathrm{RO}=$ Religiosidade Organizacional; $\mathrm{RNO}=$ Religiosidade Não Organizacional; RI= Religiosidade Intrínseca; LAF= limitação por aspectos físicos; $\mathrm{SG}=$ saúde geral; $\mathrm{AS}=$ aspectos sociais; $\mathrm{LAE}=$ limitações por aspectos emocionais; $\mathrm{SM}=$ saúde mental; *Fisher coeficiente; $\mathrm{p}$ (significância estatística $\mathrm{p} \leq 0,05)$, Cramer's $\mathrm{V}=$ coeficiente de correlação, medida de efeito.

Fonte- Pesquisa de campo. Cachoeira - BA, 2011.

\section{DISCUSSÃO}

A análise dos dados evidenciou que os idosos estudados possuem elevado índice de qualidade de vida, já que oito dos domínios de QVRS medidos pelo instrumento SF-36 apresentaram escore maior que 50 e apenas dois domínios foram menores, o de "vitalidade" $(64,4)$ e "limitação por aspectos físicos" (66,9). "Limitação por aspectos emocionais" (77,3) e "saúde mental" $(73,5)$ foram os que apresentaram maiores escores. Esses resultados se mostraram acima dos valores encontrados em outro estudo de qualidade de vida relacionada à saúde de idosos num município de São Paulo, em que os escores estiveram ao redor de 58 e mais. ${ }^{21}$
Quanto à denominação religiosa, a ESF de Capoeirucú (Cachoeira-BA) apresentou prevalência de $60,2 \%$ de católicos; $27,7 \%$ de evangélicos; e 3,6 não relataram. Segundo os dados do censo de $2010,64,99 \%$ da população brasileira referiam ser católicos; 22,16\% evangélicos e $8,04 \%$ sem religião. ${ }^{22}$

Presume-se que um fator que pode contribuir para a qualidade de vida desses idosos é que, em geral, pessoas religiosas apresentam um comportamento saudável, já que a maioria das religiões ditam normas e até mesmo o que comer e não beber. Estudos de saúde pública têm demonstrado que as pessoas religiosas têm menor probabilidade de apresentar comportamentos de 
riscos, como uso de drogas, cigarro e álcool. Isso contribui para uma saúde mais equilibrada por um período mais longo. ${ }^{23}$

Neste estudo, encontrou-se que 61\% dos idosos frequentam um templo ou igreja de uma vez por semana até uma vez por ano. Com o avançar da idade, os idosos tendem a diminuir a frequência a cultos religiosos, templos ou encontros religiosos formais, pois se deparam com limitações físicas, como dificuldade de locomoção decorrente de doenças crônicas, de sequelas de acidente vascular encefálico e da própria idade. Somam-se a isto o medo de quedas, o medo de sair desacompanhado e passar por qualquer tipo de apuro ou violência. ${ }^{24}$

Estudos têm revelado que, para cada nível de frequência à igreja, templo ou encontros religiosos, há redução progressiva no número de mortes; o comparecimento a serviços religiosos pelo menos uma vez por semana reduz em quase 50 por cento os riscos de mortes no ano seguinte. ${ }^{25}$

A participação de idosos nas atividades religiosas formais costuma diminuir com a idade, em virtude do número de doenças crônicas que os aflige. Em estudo qualitativo com 20 idosos, $25 \%$ relataram possuir pelo menos uma doença crônica; $15 \%$, duas; $40 \%$, três; $15 \%$, uma; e $5 \%$ possuíam cinco doenças. Porém, para o enfrentamento dessas doenças, principalmente aquelas associadas com a dor, os idosos se valiam da sua espiritualidade. ${ }^{26}$

Para compensar a não frequência à igreja, os idosos acabam dedicando mais tempo a atividades religiosas individuais. Neste estudo, 79,3\% deles praticam essas atividades particulares diariamente até mais de uma vez ao dia. Essa compensação à infrequência aos cultos religiosos, pela maior frequência nas atividades religiosas não formais e pelo aumento da devoção pessoal, tende a aumentar com a idade e contribui sobremaneira para a manutenção da vida. ${ }^{27}$

Em relação à RI, 95\% sentem a presença de Deus em suas vidas, 90,2\% agem conforme suas crenças e $84,2 \%$ esforçam-se para viver a religião em todos os aspectos da vida. Nesta direção, vale destacar que a RI associa-se aos aspectos psicológicos da religiosidade, ou seja, às crenças, conhecimentos e atitudes relativas à experiência religiosa bem como aos "autorrelatos" de tais experiências e ao significado pessoal atribuído à religião. ${ }^{6}$ Vivenciar a religião de maneira intrínseca significa colocar suas crenças religiosas em primeiro lugar, viver de acordo com seus preceitos, numa atitude de comprometimento e busca de sentido da vida. ${ }^{18}$

Em estudo com 560 pessoas, os aspectos qualitativos da reza e a forma de fazê-lo foram os que apresentaram maior efeito sobre a qualidade de vida. ${ }^{28} \mathrm{O}$ envolvimento religioso parece ter significativo efeito protetor para o bem-estar físico, uma vez que ter uma visão bio-psicosocioespiritual da saúde implica enxergar de maneira mais integrada a vida, reconhecendo a relação da espiritualidade com as outras dimensões, nunca se esquecendo de que o enfrentamento das situações dolorosas é importante para a melhoria da qualidade de vida e para o crescimento do ser humano. ${ }^{29}$

Os profissionais de saúde poderiam valorizar mais o cuidado relacionado à religiosidade em idosos e contribuir para melhorar sua qualidade de vida, utilizando as ferramentas de pesquisa em religiosidade e espiritualidade por meio dos instrumentos de medida validados no Brasil. ${ }^{16,30}$

Este estudo buscou associação entre qualidade de vida e religiosidade em um desenho de corte transversal; no entanto, apresenta limitações, uma vez que não se evidenciou uma relação causal entre as variáveis. $O$ tamanho da amostra também limita a abrangência da pesquisa, deixando claro que a mesma poderia ter sido realizada em outras ESFs da cidade de Cachoeira-BA.

\section{CONCLUSÃO}

Os objetivos deste estudo foram alcançados, ao ser demonstrada associação entre as dimensões da qualidade de vida relacionada à 
saúde de idosos e os vários níveis da religiosidade: organizacional, não organizacional e intrínseca.

Uma das dimensões da qualidade de vida relacionada à saúde (QVRS) que mais se associou à religiosidade organizacional, religiosidade não organizacional e religiosidade intrínseca foi a dimensão do componente mental: limitação por aspectos emocionais. Já a dimensão "saúde mental" esteve associada somente à religiosidade intrínseca. Nenhuma associação entre religiosidade e as dimensões "capacidade funcional" e "vitalidade" foi encontrada.

Notou-se a importância que os profissionais podem atribuir à atenção à saúde da pessoa idosa, avaliando as necessidades espirituais desse grupo específico, visando melhorar sua qualidade de vida relacionada à saúde.

Contatou-se, ainda, que o uso da religiosidade como recurso terapêutico é compatível com a saúde mental e física, permitindo elucidar um

\section{REFERÊNCIAS}

1. Duarte YAO, Diogo MJD. Atendimento domiciliar: um enfoque gerontológico. São Paulo: Atheneu; 2005.

2. Goldstein LL, Sommerhalder C. Religiosidade, espiritualidade e significado existencial na vida adulta e velhice. In: Freitas EV, Py L, Neri AL, Cançado FAX, Gorzoni ML, Rocha SM, editores. Tratado de geriatria e gerontologia. Rio de Janeiro: Guanabara Koogan; c2002. p. 950-64.

3. Lindolpho MC, Sá SPC, Robers LMV. Espiritualidade/Religiosidade: um suporte na assistência de enfermagem ao idoso. Em extensão 2009;8(1):117-27.

4. Levin JS, Chatters LM, Taylor RJ. A multidimensional measure of religious involvement for African Americans. Sociol Q 1995;36(1):157-73.

5. Koenig HG, McCollough ME, Larson DB. Handbook of religion and health. New York: Oxford University Press; 2001.

6. Cardoso MCS, Ferreira MC. Envolvimento religioso e bem estar subjetivos em idosos. Psicol, Ciênc Prof 2009;29(2):380-93. melhor desempenho na qualidade de vida dos idosos. Pôde-se inferir que existe associação positiva entre religiosidade nas três dimensões (RO, RNO e RI) e a qualidade de vida em pessoas idosas. Uma vez que o envelhecimento é uma realidade no Brasil e no mundo, torna-se de grande valia expandir esses resultados para a melhoria da qualidade de vida em pessoas idosas.

Ressalta-se ainda que precisam ser feitos maiores avanços em pesquisas que objetivem buscar relações científicas mais robustas para evidenciar tal associação.

\section{AGRADECIMENTO}

À Profa. Dra. Maria Dyrce Dias Meira, coordenadora do grupo de pesquisa Religiosidade/Espiritualidade na Integralidade da Saúde do Mestrado em Promoção da Saúde do Centro Universitário Adventista de São Paulo - UNASP-SP, pela revisão crítica do artigo.

7. Koenig HG, George LK, Titus P. Religion, spirituality, and health in medically ill hospitalized older patients. J Am Geriatr Soc 2004;52(4):554-62.

8. Araújo MF, De Almeida MI, Cidrack ML, Queiroz HMC, Pereira MCS, Menescal ZLC. O papel da religiosidade na promoção da saúde do idoso. Rev Bras Promoç Saúde 2008;21(3):201-8.

9. Lucchetti G, Lucchetti G, Granero AL, Bassi RM, Nasri F, Nacif SAP. O idoso e sua espiritualidade: impacto sobre diferentes aspectos do envelhecimento. Rev Bras Geriatr Gerontol 2011;14(1):159-67.

10. Saad M, Medeiros R. Espiritualidade e Saúde. Einstein (São Paulo) 2008;6(3 Pt 2):135-6.

11. Abdala GA, Rodrigues WG, Torres A, Rios MC, Brasil MS. A religiosidade/espiritualidade como influência positiva na abstinência, redução e/ou abandono do uso de drogas. REVER [Internet] 2010 [acesso em 28 ago 2013]:77-98. Disponível em: http://www.pucsp.br/rever/rv1_2010/i_abdala.htm

12. Brasil. Ministério da Saúde. Estatuto do idoso. 2 reimpr. Brasília: Ministério da Saúde; 2003. 
13. Centers for Disease Control and Prevention. Measuring healthy days: population assessment of health-related quality of life. Atlanta, Georgia: CDC; 2000.

14. Cruz KCT. Qualidade de vida relacionada à saúde dos idosos do Estudo SABE [tese]. Campinas: Universidade Estadual de Campinas; 2012.

15. Marconi MA, Lakatos EM. Fundamentos da metodologia científica. $5^{a}$ ed. São Paulo: Atlas; 2003.

16. Taunay TCD, Gondim FAA, Macêdo DS, Moreira-Almeida A, Gurgel LA, Andrade LMS, et al. Validação da versão brasileira da escala de religiosidade de Duke (DUREL). Rev Psiquiatr Clin 2012;39(4):130-35.

17. Carmona C, Claudino AD. Dimensões relacionais subjetivas na compreensão do envelhecimento em idosos. In: Actas do $7^{\circ}$ Simpósio Nacional de Investigação em Psicologia; 4-6 de fevereiro 2010; Portugal. Portugal Universidade do Minho; 2010.

18. Allport GW, Ross JM. Personal religious orientation and prejudice. J Pers Soc Psychol 1967;5(4):432-43.

19. Pimenta FAP, Simil FF, Torres HOG, Amaral CFS, Rezende CF, Coelho TO, et al. Avaliação da qualidade de vida de aposentados com a utilização do questionário SF - 36. Rev Assoc Med Bras 2008;54(1):55-60.

20. Moraes NAS, Witter GP. Velhice: qualidade de vida intríseca e extrínseca. Bol Psicol 2007;57(127):215-38.

21. Barricelli ILFOBL, Sakumoto IKY, Silva LHM, Araujo CV. Influência da orientação religiosa na qualidade de vida de idosos ativos. Rev. Bras.Geriatr. Gerontol. 2012;15(3):505-15.
22. Instituto Brasileiro de Geografia e Estatística [Internet]. Rio de Janeiro; [2010] - [acesso em 29 ago 2013]. Disponível em: http://www.sidra.ibge.gov.br/ $\mathrm{bda} / \mathrm{tabela} /$ protabl.asp? $\mathrm{c}=1489 \& \mathrm{z}=\mathrm{cd} \& \mathrm{o}=13 \& \mathrm{i}=\mathrm{P}$

23. Fleck MPA. A avaliação de qualidade de vida. Porto Alegre: Artmed; 2008.

24. Duarte FM, Wanderley KS. Religião e espiritualidade de idosos internados em uma enfermaria geriátrica. Psicol Teor Pesqui 2011;27(1):49-53.

25. Vasconcelos EM. Associação entre vida religiosa e saúde: uma breve revisão de estudos quantitativos. RECIIS 2010;4(3):12-8.

26. Ellison CG. Religious involvement and subjective well-being. J Health Soc Behav 1991;32:80-99.

27. Rocha ACAL. A espiritualidade no manejo da doença crônica do idoso [Dissertação]. São Paulo: Universidade de São Paulo, Escola de Enfermagem; 2011.

28. Paloma M, Pendleton BF. Exploring types of prayer and quality of life: a research note. Rev Relig Res 1989;31(1):46-53.

29. Da Costa CC, De Bastiani M, Geyer JG, Calvetti PU, Muller MC, De Moraes MLA. Qualidade de vida e bem estar espiritual em universitários de psicologia. Psicol estud 2008;13(2):249-55.

30. Kimura M, De Oliveira AL, Mishima LS, Underwood LG. Adaptação cultural e validação da Underwood's Daily Spiritual Experience Scale - versão brasileira. Rev Esc Enferm USP 2012;46(Esp):99-106. 